\title{
Callispheres $₫$ Drug-Eluting Beads Transarterial Chemoembolization Might be an Efficient and Safety Down-Staging Therapy in Unresectable Liver Cancer Patients
}

\section{Ning Peng}

Guangxi Medical University First Affiliated Hospital

\section{Linfeng Mao}

Guangxi Medical University First Affiliated Hospital

\section{Yiwen Tao}

Guangxi International Zhuang Medicine Hospital

\section{Kaiyin Xiao}

Guangxi Medical University First Affiliated Hospital

\section{Guandou Yuan}

Guangxi Medical University First Affiliated Hospital

\section{Songqing He ( $\nabla$ Dr_hesongqing@163.com )}

Guangxi Medical University First Affiliated Hospital https://orcid.org/0000-0002-8966-2195

\section{Research}

Keywords: Callispheres ${ }^{\circledR}$ drug-eluting beads transarterial chemoembolization, down-staging, unresectable liver cancer patients, tumor diameter, residual liver volume

Posted Date: November 1st, 2021

DOI: https://doi.org/10.21203/rs.3.rs-1012045/v1

License: (c) (i) This work is licensed under a Creative Commons Attribution 4.0 International License. Read Full License 


\section{Abstract}

Purpose: The purpose was to explore the effect of drug-eluting beads transarterial chemoembolization (DEB-TACE) on down-staging in unresectable liver cancer patients.

Methods: 15 unresectable liver cancer patients received DEB-TACE as a down-staging treatment before hepatectomy were enrolled. Data (including demographics, histories, clinical features at diagnosis and cycles of DEB-TACE before hepatectomy) were collected. Treatment response was evaluated at one month after DEB-TACE. Tumor diameter was evaluated by abdominal computed tomography scan. The residual liver volume was evaluated by IQQA liver system, Relapse-free survival (RFS) and overall survival (OS) were calculated by Kaplan-Meier curves.

Results: After DEB-TACE, 3 (20.0\%) patients achieved complete response (CR) and 10 (66.7\%) patients achieved objective response rate (ORR), meanwhile, 5 (27.8\%) tumors achieved CR and $12(66.7 \%)$ tumors achieved ORR. Tumor diameter was decreased after DEB-TACE compared to before DEB-TACE $(9.4 \pm 3.3 \mathrm{vs}$. $5.4 \pm 3.5 \mathrm{~cm})(P<0.01)$. As to residual liver volume, it was increased after DEB-TACE compared to before DEB-TACE $\left(1066.2 \mathrm{~cm}^{3}\right.$ vs. $\left.1172.5 \mathrm{~cm}^{3}\right)(P=0.007)$. More importantly, $15(100 \%)$ patients could receive resection after DEB-TACE, (including 14 (93.3\%) patients with curative resection and 1 (6.7\%) patient with palliative resection). For survival, the median RFS was 26.0 months, and the percentage of 5-year accumulating RFS was $20 \%$. As to OS, the median OS was 54.5 months, and the percentage of 5-year accumulating OS was $40 \%$. For safety profiles, 5 patients had postoperative pain, 7 patients had fever, and 1 had nausea and vomiting.

Conclusion: DEB-TACE might be an efficient and safety down-staging treatment in unresectable liver cancer patients.

\section{Introduction}

Liver cancer is a kind of complex disease frequently arising in the setting of chronic liver disease and cirrhosis, which is considered as the sixth common diagnosed cancer and the second leading cause of death from cancer around the world, resulting in 841080 new cases and 781631 deaths during 2018 globally ${ }^{[1]}$. Despite the widespread use of surveillance programs (such as hepatic resection, liver transplantation and image-guided tumor ablation), not all liver cancer patients could get benefits. For instance, hepatic resection is the optimally curable choice for liver cancer patients, particular in these patients at early stage, whereas most patients diagnosed as liver cancer are at intermediate or advanced stages and lost the best time to receive surgery due to unbearable invasion ${ }^{[2,3]}$. Although liver transplantation is another curable treatment for patients with small multinodular tumors or advanced liver dysfunction, it is still limitedly applicated owing to the shortage of donators and strict blood type matching requirements ${ }^{[2]}$. In regard to image-guided tumor ablation, its most procedures are done percutaneously, and it could achieve complete necrosis of almost $100 \%$ in liver tumors smaller than $2 \mathrm{~cm}$, while less effectiveness occurs in larger tumors ${ }^{[2,4,5]}$. 
For patients with unresectable liver cancer clinically, transarterial chemoembolization (TACE) is widely regarded as first-line therapy for sake of reduced collateral damage to tumor-free parenchyma and increased treatment effect, which is divided into two types according to different chemotherapy modalities (including conventional TACE (CTACE) and drug-eluting beads TACE (DEB-TACE)) ${ }^{[6]}$. In detail, CTACE is a kind of conventional technology using lipiodol as chemotherapy drug carriers, while it has been determined to have high systemic toxicity ${ }^{[7]}$. So as to solve this problem, DEB-TACE has been introduced, which is a novel drug-delivery embolization system using microspheres as embolic agents and loading with chemotherapeutic drugs to gradually release them into the target tumor. Despite the benefits of DEB-TACE with accurate drug delivery and permanent vascular embolization, it is still not a curative treatment, and its disadvantages also contain liver function deterioration and incomplete tumor necrosis ${ }^{[8]}$. Therefore, convincing treatments combining with the advantages of DEB-TACE and other curable therapy are needed to be explored. Taken together with the above mentioned, we hypothesized DEB-TACE has down-staging effects for unresectable liver cancer patients, and after down staging, these patients could receive curable hepatic resection. However, little is known about the down-staging benefit in unresectable liver cancer patients. In an attempt to address this dilemma, we carried out this study with the purpose of investigating the effect of DEB-TACE on down-staging in unresectable liver cancer patients.

\section{Methods}

\section{Patients selection}

From May 2016 to June 2018, 15 unresectable liver cancer patients who received DEB-TACE as downstaging treatment before hepatectomy in The First Affiliated Hospital of Guangxi Medical University were enrolled in this study. The screening criteria were as follows: (i) diagnosed as primary hepatocellular carcinoma (HCC) or cholangiocarcinoma by postoperative pathological findings in accordance with American Association for the Study of the Liver Diseases (AASLD) guidelines, (ii) unable to receive resection due to the following reasons: (a) presenting with large and multiple tumors and insufficient residual liver; (b) presenting with poor liver function and potential risk of severe post-excision cirrhosis, incomplete compensation of residual liver function and post-excision liver failure; (c) coronary heart disease or other severe complications resulting in a higher surgical risk; (iii) The number of tumors $\leq 2$, which were confined to the half liver and had no main portal vein invasion, underwent DEB-TACE as downstaging therapy, (iv) had no history of treatment for liver cancer before DEB-TACE, (v) clinical data and follow-up documents were complete, (vi) not complicated with other malignancies. The approval for this study was acquired from the Institutional Review Board of The First Affiliated Hospital of Guangxi Medical University, and the written informed consent or verbal agreement with tape recording was obtained from enrolled patients or their guardians.

\section{Baseline Data Collection}


Data of enrolled patients were collected from medical documents, including (1) demographics (age, and gender), (2) histories (drink, hepatitis B (HB), hepatic encephalopathy, hypertension, heart disease, and liver cirrhosis), (3) clinical features at diagnosis (histological type, number of tumors, largest tumor size, portal vein invasion, lymphadenectasis, distant metastasis, liver involvement, Eastern Cooperative Oncology Group (ECOG) score, Barcelona clinic liver cancer (BCLC) stage, and Child-Pugh stage), (4) cycles of DEB-TACE before hepatectomy.

\section{Treatment Process}

The CalliSpheres ${ }^{\circledR}$ Microspheres (CSM) (Jiangsu Hengrui Medicine Co. Ltd., Jiangsu, China) were used as drug-eluting beads in the present study, which had the diameter ranging from $100 \mu \mathrm{m}$ to $300 \mu \mathrm{m}$. Before operation, the CSM was loaded with pirarubicin $(20 \sim 40 \mathrm{mg})$, which was prepared as previously described

[9]. The DEB-TACE operation was conducted in the digital subtraction angiography (DSA) room. Briefly, the tumor supplying vessels were identified by the hepatic angiography using segment or subsegment super selective catheterization, and the femoral artery was punctured using microcatheter, which were performed in accordance with previous study ${ }^{[10]}$. When the microcatheter was precisely inserted into the tumor supplying vessel, $50 \mathrm{mg}$ lobaplatin, $500 \mathrm{mg}$ fluorouracil as well as $10 \mathrm{~mL}$ lipiodol were successively infused into the tumor supplying vessel, then, a bottle of CSM loading with pirarubicin were infused into the tumor supplying vessel. For patients with huge tumor, 1 3 bottle(s) of blank CSM (not loaded with drugs) were added additionally for embolization following the infusion of CSM loading with pirarubicin. Finally, a spot of lipiodol was injected into the tumor supplying vessel for preventing reverse flow of microspheres, resulting in a "sandwich" effect. Besides, pre-procedure and post-procedure treatments were carried out in line with the previous study ${ }^{[10]}$. With respect to the patients presenting limited efficacy by one cycle of DEB-TACE, if necessary, repeated DEB-TACE was administered for them. Finally, all patients underwent curative resection or palliative resection, which was depended on the efficacy of down-staging treatment by DEB-TACE.

\section{Assessments}

The sum of the largest diameters of target tumors in arterial enhancement on Computed Tomography (CT), the residual liver volume, the BCLC stage, the Child-Pugh stage, the tumor markers (including alpha fetoprotein (AFP), carcino-embryonic antigen (CEA) and carbohydrate antigen199 (CA199)) as well as the liver indexes (albumin (ALB), total protein (TP), total bilirubin (TBIL), total bile acid (TBA), alanine aminotransferase (ALT), aspartate aminotransferase (AST), and alkaline phosphatase (ALP)) were examined and documented before and after down-staging treatment by DEB-TACE. The response to DEBTACE therapy was evaluated at one month after DEB-TACE by enhanced CT or magnetic resonance imaging (MRI) examination, and the response criteria were in line with the modified Response Evaluation Criteria in Solid Tumors (mRECIST), as follows: (1) complete response (CR): disappearance of any intratumoral arterial enhancement in all target lesions; (2) partial response (PR): at least a $30 \%$ decrease 
in the sum of diameters of viable (enhancement in the arterial phase ) target lesions; (3) stable disease (SD): any cases that did not qualify either PR or progressive disease (PD); (4) PD: an increase of at least $20 \%$ in the sum of the diameters of the viable (enhancing) target lesions. Further, objective response rate (ORR) was defined as CR+PR. In addition, the patients' surgical type (curative resection or palliative surgery) was also recorded, and the adverse events occurred post DEB-TACE therapy were documented as well.

\section{Follow-up}

After surgery, patients were regularly followed up by clinic visits or telephone calls. The last follow-up date was 2021/09/31. The total follow-up duration was ranging from 12 to 38 months, and median follow-up duration was 23.2 months. Relapse-free survival (RFS) was calculated from the date of surgical resection to the date of disease relapse or death, whichever occurred first. Overall survival (OS) was calculated from the date of DEB-TACE therapy to the date of death or last follow-up.

\section{Statistical analysis}

The residual liver volume was evaluated by IQQA liver system (EDDA Technology, Inc, New Jersey, USA). Statistical data processing was conducted on SPSS 24.0 software (SPSS Inc, Chicago, USA), and the figure construction was performed on GraphPad Prism 7.01 software (GraphPad Software Inc, San Diego, USA). Continuous data were described as mean and standard deviation (SD), or median and interquartile range (IQR); categorical data were displayed as number (percentage). Comparison of the sum of target tumor largest diameters and residual liver volume before and after down-staging treatment by DEB-TACE was determined by paired-sample t test; Comparison of the BCLC stage, the Child-Pugh stage before and after down-staging treatment by DEB-TACE was determined by McNemar's test; Comparison of tumor markers and liver indexes before and after down-staging treatment by DEB-TACE was determined by Wilcoxon signed-rank test. RFS and OS were illustrated using Kaplan-Meier curves. $P$ value $<0.05$ was considered significant.

\section{Results}

\section{Patients' characteristics}

There were 15 unresectable liver cancer patients (including 12 (80.0\%) males and 3 (20.0\%) females) with the mean age of $48.9 \pm 14.2$ years old (Table 1 ). For clinical features, the mean value of largest tumor diameter was $8.8 \pm 3.9 \mathrm{~cm}$. The numbers of patients with ECOG score at 0,1 , and 2 were $2(13.3 \%), 11$ (73.4\%) and $2(13.3 \%)$ respectively. Based on BCLC stage, 8 (53.3\%) patients were at A stage, $3(20.0 \%)$ at B stage, and 4 (26.7) at C stage. According to Child-Pugh stage, 14 (93.3\%) patients were at A stage, and 1 $(6.7 \%)$ patient was at B stage. In addition, there were 10 (66.6\%) patients received 1 cycle of DEB-TACE, 4 
(26.7\%) patients received 2 cycles of DEB-TACE, and 1 (6.7\%) patients received 3 cycles of DEB-TACE. The detailed information about others clinical characteristics of patients were shown in Table 1. 
Table 1

Clinical characteristics of patients

\section{Items}

Age (years), mean $\pm S D$

Gender, No. (\%)

Male

Female

History of drink, No. (\%)

History of HB, No. (\%)

History of hepatic encephalopathy, No. (\%)

History of hypertension, No. (\%)

History of heart disease, No. (\%)

Liver cirrhosis, No. (\%)

Histological type, No. (\%)

$\mathrm{HCC}$

Cholangiocarcinoma

Number of tumors, No. (\%)

1

2

Largest tumor diameter $(\mathrm{cm})$, mean $\pm S D$

Portal vein invasion, No. (\%)

Lymphadenectasis, No. (\%)

Distant metastasis, No. (\%)

Liver involvement, №. (\%)

$\leq 50 \%$

$50-70 \%$

$\geq 70 \%$

Unknown
Patients $(\mathrm{N}=15)$

$48.9 \pm 14.2$

$12(80.0)$

$3(20.0)$

5 (33.3)

$12(80.0)$

2 (13.3)

$3(20.0)$

1 (6.7)

$9(60.0)$

$13(86.7)$

$2(13.3)$

$12(80.0)$

$3(20.0)$

$8.8 \pm 3.9$

1 (6.7)

$5(33.3)$

$2(13.3)$

$1(6.7)$

10 (66.7)

$3(20.0)$

1 (6.7)

SD, standard deviation; $\mathrm{HB}$, hepatitis $\mathrm{B}$; $\mathrm{HCC}$, hepatocellular carcinoma; ECOG, Eastern Cooperative Oncology Group; BCLC, Barcelona clinic liver cancer; DEB-TACE, drug-eluting bead transarterial chemoembolization. 
ECOG score, No. (\%)

$\begin{array}{ll}0 & 2(13.3) \\ 1 & 11(73.4) \\ 2 & 2(13.3)\end{array}$

BCLC stage, No. (\%)

A

B

$3(20.0)$

C

4 (26.7)

Child-Pugh stage, No. (\%)

A

14 (93.3)

B

$1(6.7)$

Cycles of DEB-TACE, No. (\%)

SD, standard deviation; HB, hepatitis B; HCC, hepatocellular carcinoma; ECOG, Eastern Cooperative Oncology Group; BCLC, Barcelona clinic liver cancer; DEB-TACE, drug-eluting bead transarterial chemoembolization.

\section{Treatment Response After Deb-tace}

After DEB-TACE treatment at one month, in total patients, 3 (20.0\%) patients achieved CR, 7 (46.7\%) patients achieved PR and 10 (66.7\%) patients achieved ORR (Figure 1A). As for PR patients, 2 (28.6\%) patients presented with necrosis rate $<50 \%, 4(57.1 \%)$ patients presented with necrosis rate of $50-80 \%$, and $1(14.3 \%)$ patient presented with necrosis rate $>80 \%$ (Figure 1B). In total tumors, 5 (27.8\%) tumors achieved CR, 7 (38.9\%) tumors achieved PR and 12 (66.7\%) tumors achieved ORR (Figure 1C). As for PR tumors, $2(28.6 \%)$ tumors presented with necrosis rate $<50 \%, 4(57.1 \%)$ tumors presented with necrosis rate of $50-80 \%$, and $1(14.3 \%)$ tumors presented with necrosis rate $>80 \%$ (Figure 1D). 
Comparison of tumor diameters, BCLC stage and Child-Pugh stage before and after DEB-TACE

For tumor diameters, it was decreased after DEB-TACE compared to before DEB-TACE $(9.4 \pm 3.3 \mathrm{vs.5.4 \pm 3.5}$ $\mathrm{cm})(P<0.01)$ (Figure 2A). However, there was no difference in BCLC stage $(\mathrm{P}=0.317)$ (Figure 2B) and Child-Pugh stage (Figure 2C).

\section{Comparison of the residual liver volume before and after DEB-TACE}

The mean value of residual liver volume before and after DEB-TACE was $1066.2 \mathrm{~cm}^{3}$ and $1172.5 \mathrm{~cm}^{3}$ respectively (Table 2). Compared to before DEB-TACE, residual liver volume was increased after DEB-TACE (mean value of increased rate was $11.4 \%)(P=0.007)$. The detailed information about the residual liver volume in each patient before and after DEB-TACE was shown in Table 2. 
Table 2

The residual liver volume before and after DEB-TACE

\begin{tabular}{|c|c|c|c|c|c|}
\hline \multirow[t]{2}{*}{ No. } & \multicolumn{4}{|c|}{ Residual liver volume $\left(\mathrm{cm}^{3}\right)$} & \multirow[t]{2}{*}{$P$ value } \\
\hline & Before DEB-TACE & After DEB-TACE & Increase & Increase rate (\%) & \\
\hline 1 & 1182.0 & 1064.0 & -118.0 & -10.0 & \multirow[t]{16}{*}{0.007} \\
\hline 2 & 1006.0 & 1083.0 & 77.0 & 7.7 & \\
\hline 3 & 1266.0 & 1407.0 & 141.0 & 11.1 & \\
\hline 4 & 1021.8 & 972.8 & -49.0 & -4.8 & \\
\hline 5 & 705.0 & 832.0 & 127.0 & 18.0 & \\
\hline 6 & 1514.0 & 1659.0 & 145.0 & 9.6 & \\
\hline 7 & 796.8 & 706.0 & -90.8 & -11.4 & \\
\hline 8 & 784.0 & 1169.0 & 385.0 & 49.1 & \\
\hline 9 & 1547.0 & 1740.0 & 193.0 & 12.5 & \\
\hline 10 & 1266.0 & 1358.0 & 92.0 & 7.3 & \\
\hline 11 & 1371.0 & 1371.0 & 0.0 & 0.0 & \\
\hline 12 & 1127.0 & 1347.0 & 220.0 & 19.5 & \\
\hline 13 & 944.0 & 1066.0 & 122.0 & 12.9 & \\
\hline 14 & 909.0 & 1100.0 & 191.0 & 21.0 & \\
\hline 15 & 554.0 & 713.0 & 159.0 & 28.7 & \\
\hline Mean & 1066.2 & 1172.5 & 106.3 & 11.4 & \\
\hline
\end{tabular}

\section{Comparison Of Liver Function Indexes Before And After Deb-tace}

No difference was found in liver function indexes, including ALB $(P=0.334)$, TP $(P=0.256)$, TBIL $(P=0.733)$, TBA $(P=0.513)$, ALT $(P=0.609)$ and AST $(P=0.593)$ and ALP $(P=0.050)$ (Table 3$)$. The detailed information was shown in Table 3. 
Table 3

The level of liver function indexes before and after DEB-TACE

\begin{tabular}{|llll|}
\hline Liver function indexes & Before DEB-TACE & After DEB-TACE & Pvalue \\
\hline ALB $(\mathrm{g} / \mathrm{L})$ & $42.4(38.2-44.7)$ & $39.8(37.0-42.3)$ & 0.334 \\
\hline TP $(\mathrm{g} / \mathrm{L})$ & $70.5(63.2-76.0)$ & $72.0(63.8-79.0)$ & 0.256 \\
\hline TBIL $(\mu \mathrm{mol} / \mathrm{L})$ & $8.7(7.3-10.9)$ & $10.2(7.0-13.0)$ & 0.733 \\
\hline TBA $(\mu \mathrm{mol} / \mathrm{L})$ & $7.3(2.9-15.9)$ & $6.2(3.4-13.9)$ & 0.513 \\
\hline ALT $(\mathrm{U} / \mathrm{L})$ & $50.0(22.0-59.0)$ & $30.0(24.0-46.0)$ & 0.609 \\
\hline AST $(\mathrm{U} / \mathrm{L})$ & $33.0(26.0-59.0)$ & $30.0(26.0-37.0)$ & 0.593 \\
\hline ALP $(\mathrm{U} / \mathrm{L})$ & $109.0(91.0-130.0)$ & $120.0(94.0-158.0)$ & 0.050 \\
\hline $\begin{array}{l}\text { Data were displayed as median (interquartile range). Comparison was determined by Wilcoxon signed- } \\
\text { rank test. DEB-TACE, drug-eluting bead transarterial chemoembolization; ALB, albumin; IQR, } \\
\text { interquartile range; TP, total protein; TBIL, total bilirubin; TBA, total bile acid; ALT, alanine transaminase; } \\
\text { AST, aspartate aminotransferase; ALP, alkaline phosphatase. }\end{array}$ \\
\hline
\end{tabular}

\section{Comparison Of Tumor Markers Before And After Deb-tace}

For tumor markers, no difference was discovered in AFP $(P=0.300)$ (Figure 3A), CEA $(P=0.505)$ (Figure 3B) and CA191 ( $P=0.091)$ (Figure 3C).

\section{Operation Types}

In total patients, there were $14(93.3 \%)$ patients received with curative resection, and 1 (6.7\%) patient received palliative resection (Figure 4).

\section{Rfs And Os}

The median value of RFS was 26.0 months, and the percentage of 5-year accumulating RFS was $20 \%$ (Figure $5 \mathrm{~A}$ ). As to OS, the median value of OS was 54.5 months, and the percentage of 5-year accumulating OS was $40 \%$ (Figure $5 \mathrm{~B}$ ).

\section{Adverse Events}

After DEB-TACE, there were 5 patients presented with pain (NRS score ranging from 5 to 9), 7 patients presented with fever and 1 patient presented with nausea and vomiting (Table 4). The detailed information was shown in Table 4. 
Table 4

Adverse events after DEB-TACE

\begin{tabular}{|c|c|c|c|c|c|c|c|}
\hline No. & Pain & $\begin{array}{l}\text { NRS } \\
\text { score }\end{array}$ & Treatment & Fever & Treatment & $\begin{array}{l}\text { Nausea } \\
\text { and } \\
\text { vomiting }\end{array}$ & Treatment \\
\hline 1 & No & N/A & $\mathrm{N} / \mathrm{A}$ & $39.0^{\circ} \mathrm{C}$ & Ibuprofen & No & $\mathrm{N} / \mathrm{A}$ \\
\hline 2 & No & N/A & $\mathrm{N} / \mathrm{A}$ & $39.4^{\circ} \mathrm{C}$ & Ibuprofen+indometacin & No & $\mathrm{N} / \mathrm{A}$ \\
\hline 3 & No & N/A & $\mathrm{N} / \mathrm{A}$ & $39.0^{\circ} \mathrm{C}$ & Ibuprofen+indometacin & No & $\mathrm{N} / \mathrm{A}$ \\
\hline 4 & Yes & 7 & Flurbiprofen & No & N/A & No & $\mathrm{N} / \mathrm{A}$ \\
\hline 5 & No & $\mathrm{N} / \mathrm{A}$ & $\mathrm{N} / \mathrm{A}$ & No & N/A & No & $\mathrm{N} / \mathrm{A}$ \\
\hline 6 & No & N/A & $\mathrm{N} / \mathrm{A}$ & $39.0^{\circ} \mathrm{C}$ & Ibuprofen & No & $\mathrm{N} / \mathrm{A}$ \\
\hline 7 & Yes & 8 & Pethidine & No & N/A & No & $\mathrm{N} / \mathrm{A}$ \\
\hline 8 & Yes & 8 & Pethidine & $38.6^{\circ} \mathrm{C}$ & Ibuprofen+indometacin & No & $\mathrm{N} / \mathrm{A}$ \\
\hline 9 & Yes & 5 & Tramadol & $38.0^{\circ} \mathrm{C}$ & Ibuprofen & No & $\mathrm{N} / \mathrm{A}$ \\
\hline 10 & No & N/A & $\mathrm{N} / \mathrm{A}$ & No & N/A & No & $\mathrm{N} / \mathrm{A}$ \\
\hline 11 & No & $\mathrm{N} / \mathrm{A}$ & $\mathrm{N} / \mathrm{A}$ & No & N/A & No & $\mathrm{N} / \mathrm{A}$ \\
\hline 12 & Yes & 6 & Tramadol & No & $\mathrm{N} / \mathrm{A}$ & No & $\mathrm{N} / \mathrm{A}$ \\
\hline 13 & No & N/A & $\mathrm{N} / \mathrm{A}$ & No & N/A & No & $\mathrm{N} / \mathrm{A}$ \\
\hline 14 & No & N/A & $\mathrm{N} / \mathrm{A}$ & No & N/A & Yes & Palonosetron \\
\hline 15 & Yes & 9 & Pethidine & $38.4^{\circ} \mathrm{C}$ & Ibuprofen & No & $\mathrm{N} / \mathrm{A}$ \\
\hline
\end{tabular}

\section{Typical Case Report}

Mr. Huang, a 56-year-old man was first diagnosed as HCC. Before DEB-TACE treatment, abdominal computed tomography (CT) scan showed that tumor size was $80.49 \mathrm{~mm} * 110.82 \mathrm{~mm}$ (Figure 6A). After identifying the tumor supply artery by hepatic arteriography, DEB-TACE treatment was performed (Figure $6 \mathrm{~B})$. After the operation, hepatic arteriography was carried out immediately, which disclosed that the tumor was reduced and the tumor blood supply arteries were almost stagnant (Figure 6C).

Before the surgery, abdominal CT scan showed that tumor size was $40.22 \mathrm{~mm} * 64.38 \mathrm{~mm}$, which diameter was reduced nearly $5 \mathrm{~cm}$ (Figure 6D). After resection (Figure 6E, F), the pathological examination was performed (Figure 6G), which discovered drug-loaded microspheres (Figure 6H). This patient was pathologically confirmed HCC with a trend of differentiation of bile duct cell carcinoma (Figure 6I). 


\section{Discussion}

DEB-TACE is a new type of embolization material involving the injection of a chemotherapeutic agent selectively into the feeding arteries of the tumor to not only potentially obtain higher intratumor drug concentrations and maintain lower plasma drug concentrations compared with CTACE, but also block the blood vessel effectively causing infarction and necrosis ${ }^{[8]}$. Accumulating evidence reveals that the 1 month CR rate ranges from $19.2-70.6 \%$ in tumors, and ORR ranges from $56.3-100 \%$ in tumors ${ }^{[11,12]}$. In line with these previous data, we discovered that the 1-month CR rate and ORR in patients were $20.0 \%$ and $66.7 \%$, respectively, while in tumors were $27.8 \%$ and $66.7 \%$, respectively.

Based on the management guidelines published by the American Association for the Study of Liver Diseases, curative treatments have been only recommended to liver cancer patients who are with a single nodule $<5 \mathrm{~cm}$ in diameter, three or fewer nodules $<3 \mathrm{~cm}$ in diameter, or meet the Milan criteria ${ }^{[13,14]}$. Although DEB-TACE is related to promising efficacy and low toxicity in unresectable liver cancer patients, which could effectively delay tumor progression or prevent recurrence during short-term (within 6 months), it is still less effective over longer periods, and could not achieve response rates and cure the tumor comparable to curative therapy ${ }^{[15]}$. There is therefore a requirement with additional and effective treatment strategies for unresectable liver cancer patients, including the optimization of DEB-TACE and its combination with other treatment modalities. TACE has been regarded as preoperative neoadjuvant chemotherapy to improve outcomes in patients with resectable hepatocellular carcinoma (HCC), which also is referred to as "bridging" therapy before liver transplantation for $\mathrm{HCC}^{[16,17]}$. However, limited information about the down-staging effect of DEB-TACE in unresectable liver cancer patients. One previous study enrolling 30 unresectable liver cancer patients listed to liver transplantation discloses that after 30 times DEB-TACE successfully, 76.7\% patients are down-staged to meet University of California, San Francisco (UCSF) criteria and 53.3\% patients are down-staged to meet Milan criteria, meanwhile, there are 13 patients had been given liver transplantation successfully ${ }^{[18,19]}$.

Considering the advantage of DEB-TACE, we hypothesized DEB-TACE might be used as a neo-adjuvant treatment for unresectable liver cancer patients, which might have down-staging effects for those patients, and made them receive curative treatment after DEB-TACE treatment. Whereas there was no evidence about whether DEB-TACE as down-staging therapy in unresectable liver cancer patients listed to receive surgery. Thus, we performed this study and discovered that DEB-TACE decreased tumor diameter in unresectable liver cancer patients, meanwhile, we found that DEB-TACE promoted the growth of residual liver volume in unresectable liver cancer patients. The possible explanations were that DEB-TACE is not only related to a high and sustained chemotherapy drug concentration, but also benefited for embolization of the small artery supplying tumor, which guaranteed the efficacy of DEB-TACE treatment on killing tumor cells and blocking tumor blood supply to promote tumor necrosis and inhibit tumor growth, thereby contributing to down-staging effect in unresectable liver cancer patients.

More importantly, we discovered that 15 (100\%) patients could receive resection after DEB-TACE, suggesting that after DEB-TACE, the rate for unresectable patients received resection was $100 \%$. The 
probable causes were as follows: DEB-TACE presented with great efficiency in killing tumor cells and causing tumor avascular necrosis to devote into down-staging effect in unresectable liver cancer patients (above-mentioned), which made these patients have chance to receive resection again. In addition, we found the percentage of 1-year accumulating RFS was $64.6 \%$ as well as 2-year accumulating RFS was $55.4 \%$. And the percentage of 1-year accumulating OS was $100.0 \%$ and 2 -year accumulating OS was $77.8 \%$. The median value of RFS was 26.0 months, and the percentage of 5-year accumulating RFS was $20 \%$. As to OS, the median value of OS was 54.5 months, and the percentage of 5 -year accumulating OS was $40 \%$. These results were in line with a previous Chinese study disclosing the percentage of 1 -year accumulating RFS of $92.3 \%$ and the percentage of 1 -year accumulating 0 S of $100.0 \%{ }^{[19]}$.

Like each invasive treatment, DEB-TACE undertakes the risk of AEs, including abdominal pain, fever, vomiting, and increased blood pressure ${ }^{[20]}$. In this study, we also discovered that 5 cases occurred pain, 7 cases occurred fever as well as nausea, and 1 case occurred vomiting, These results indicated that there were no serious complications occurring in these unresectable liver cancer patients after DEB-TACE, which showed the advantage of DEB-TACE with low systemic toxicity and good safety.

There were still several limitations in this study. The main limitation was a relatively small sample size of 15 unresectable liver cancer patients, which might lead to poor statistical power. Further validation is necessary in a larger sample size. In addition, the relatively short follow-up duration existed in this study, hence, long-term efficacy and safety were not able to be evaluated. Furthermore, all unresectable liver cancer patients were from one hospital, there might be selected bias. Further study with more patients from a multicenter is necessary.

In summary, DEB-TACE decreases tumor diameter and promotes the growth of residual liver volume in unresectable liver cancer patients. More importantly, after DEB-TACE treatment, $100 \%$ of patients could receive surgery. These data suggest that DEB-TACE might be effective and safety as a down-staging therapy in unresectable liver cancer patients.

\section{Abbreviations}

DEB-TACE

Drug-eluting beads transarterial chemoembolization

RFS

Relapse-free survival

OS

Overall survival

$\mathrm{CR}$

Complete response

ORR

Objective response rate

$\mathrm{HCC}$ 
Hepatocellular carcinoma

PR

Partial response

CT

Computed Tomography

AFP

alpha fetoprotein

CEA

Carcino-embryonic antigen

CA199

Carbohydrate antigen199

ALB

Albumin

TP

Total protein

TBIL

Total bilirubin

TBA

Total bile acid

ALT

Alanine aminotransferase

AST

Aspartate aminotransferase

ALP

Alkaline phosphatase

MRI

Magnetic resonance imaging.

\section{Declarations}

\section{Authors' contributions}

N.P. and K.X. conceived this study, S.H. directed the study. N.P. and K.X. performed most of the experiments. N.P. drafted the manuscript. N.P., L.M. and Y.T. analyzed the data and performed the statistical analyses. L.M., G.Y. and Y.T.participated in some experiments. S.H., N.P., K.X. and L.M. provided critical intellectual revision. S.H. provided financial support.

\section{Funding and Acknowledgement}

This study was supported by the National Natural Science Foundation of China (No. 81771674, No. 91949122 and No. 82160500), the 111 Project (D17011), Guangxi BaGui Scholars (Songqing He), 
Guangxi Key Research and Development Plan (2018AD03001), and Special project of central government guiding local science and technology development (ZY20198011). Key Laboratory of Early Prevention and Treatment for Regional High Frequency Tumor (Gaungxi Medical University), Ministry of Education 『GKE-ZZ202143凶.

\section{Availability of data and materials}

Datasets used and/or analyzed during the current study are available from the corresponding author on reasonable request.

\section{Ethics approval and consent to participate}

The study was approved by the Institutional Review Board of The First Affiliated Hospital of Guangxi Medical University.

\section{Consent for publication}

Not applicable

\section{Conflict of interest}

The authors declare no potential conflicts of interest.

\section{References}

1. MOHAMMADIAN M, BAKESHEI K A, MOHAMMADIAN-HAFSHEJANI A. International epidemiology of liver cancer: geographical distribution, secular trends and predicting the future [J]. Journal of Preventive Medicine and Hygiene, 61(2):

2. FORNER A, LLOVET J M, BRUIX J. Hepatocellular carcinoma [J]. The Lancet, 2012, 379(9822): 124555 .

3. ALEJANDRO F, MAR A R, JORDI B. Hepatocellular carcinoma [J]. Lancet 2018 Mar 31;391(10127):1301-1314. doi: 10.1016/S0140-6736(18)30010-2.

4. LENCIONI R, CROCETTI L. Image-guided ablation for hepatocellular carcinoma [J]. Recent results in cancer research Fortschritte der Krebsforschung Progrès dans les recherches sur le cancer, 2013, 190(181-94.

5. BREEN D J, LENCIONI R. Image-guided ablation of primary liver and renal tumours [J]. Nature Reviews Clinical Oncology, 2015, 12(3): 175-86.

6. NORIHIRO, IMAI, MASATOSHI, et al. Transarterial chemoembolization for hepatocellular carcinoma: A review of techniques [J]. World J Hepatol. 2014 Dec 27;6(12):844-50.doi: 10.4254/wjh.v6.i12.844.

7. NI J Y, XU L F, WANG W D, et al. Conventional transarterial chemoembolization vs microsphere embolization in hepatocellular carcinoma: A meta-analysis [J]. World J Gastroenterol, 2014, 20(45): 17206-17.

8. CHEN P, YUAN P, CHEN B, et al. Evaluation of drug-eluting beads versus conventional transcatheter arterial chemoembolization in patients with unresectable hepatocellular carcinoma: A systematic 
review and meta-analysis [J]. Clinics \& Research in Hepatology \& Gastroenterology, 2016, S2210740116300900.

9. ZHOU G H, HAN J, SUN J H, et al. Efficacy and safety profile of drug-eluting beads transarterial chemoembolization by CalliSpheres beads in Chinese hepatocellular carcinoma patients [J]. BMC Cancer, 2018, 18(1): 644-.

10. XIN, ZHANG, XIAO, et al. An investigation of efficacy, safety, and prognostic factors of drug-eluting beads-transarterial chemoembolization operation with CalliSpheres? Microspheres in treating Chinese hepatocellular carcinoma patients [J]. Journal of clinical laboratory analysis, 2019, 33(8): e22975.

11. NANI, TREVISANI, GASPARINI, et al. Randomised controlled trial of doxorubicin-eluting beads vs conventional chemoembolisation for hepatocellular carcinoma [J]. The British journal of cancer, 2014,

12. SACCO R, BARGELLINI I, BERTINI M, et al. Conventional versus Doxorubicin-eluting Bead Transarterial Chemoembolization for Hepatocellular Carcinoma [J]. Journal of Vascular \& Interventional Radiology, 2011, 22(11): 1545-52.

13. BRUIX J, SHERMAN M. Management of hepatocellular carcinoma: An update [J]. Hepatology, 2011,

14. MAZZAFERRO V, REGALIA E, DOCI R, et al. Liver transplantation for the treatment of small hepatocellular carcinomas in patients with cirrhosis [J]. N Engl J Med, 1996, 334(11): 693-9.

15. JIN-WOO L, HYE J J, JIN Y J, et al. Transarterial chemoembolization for hepatocellular carcinoma: an evidence-based review of its place in therapy [J]. Journal of Hepatocellular Carcinoma, 2015, 2(123-9.

16. NISHIKAWA, HIROKI. Effect of transcatheter arterial chemoembolization prior to surgical resection for hepatocellular carcinoma [J]. International Journal of Oncology, 2013,

17. FUJIKI M, AUCEJO F, KIM R. General overview of neo-adjuvant therapy for hepatocellular carcinoma before liver transplantation: necessity or option? [J]. Liver International, 2011, 31(8): 1081-9.

18. ABDELFATTAH M R, EL-SIESY H, AL-MANEA H, et al. Liver transplantation for hepatocellular carcinoma within the Milan criteria versus the University of California San Francisco criteria: a comparative study [J]. Eur J Gastroenterol Hepatol, 2018, 30(4): 1.

19. CAI L, LI H, GUO J, et al. Drug-eluting bead transarterial chemoembolization is an effective downstaging option for subsequent radical treatments in patients with hepatocellular carcinoma: $\mathrm{A}$ cohort study [J]. Gastroentérologie Clinique et Biologique,

20. ZOU J H, ZHANG L, REN Z G, et al. Efficacy and safety of cTACE versus DEB-TACE in patients with hepatocellular carcinoma: a meta-analysis [J]. Journal of Digestive Diseases, 2016, 17(8):

\section{Figures}


A

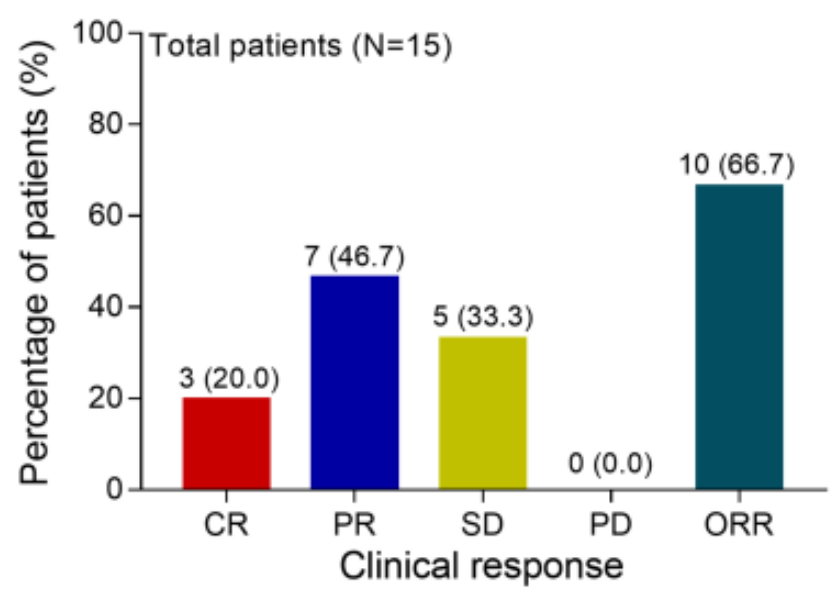

C

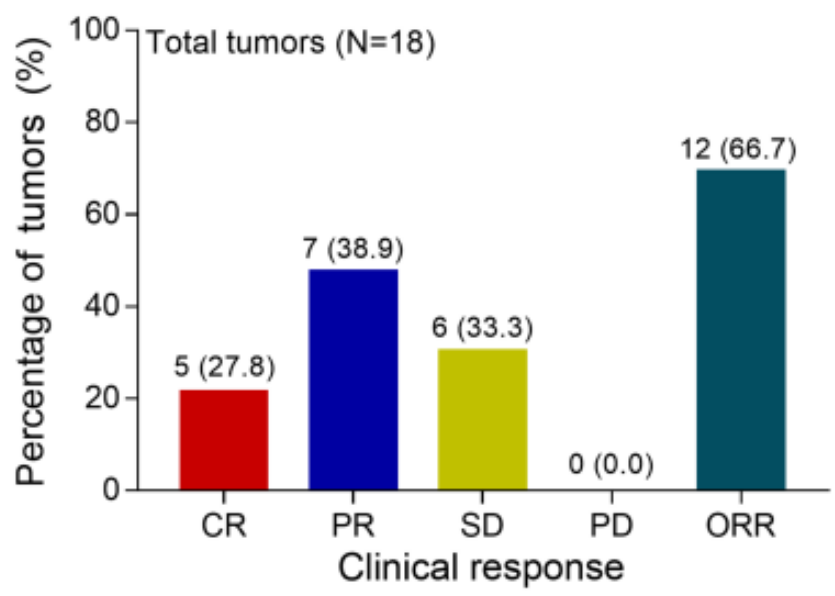

B

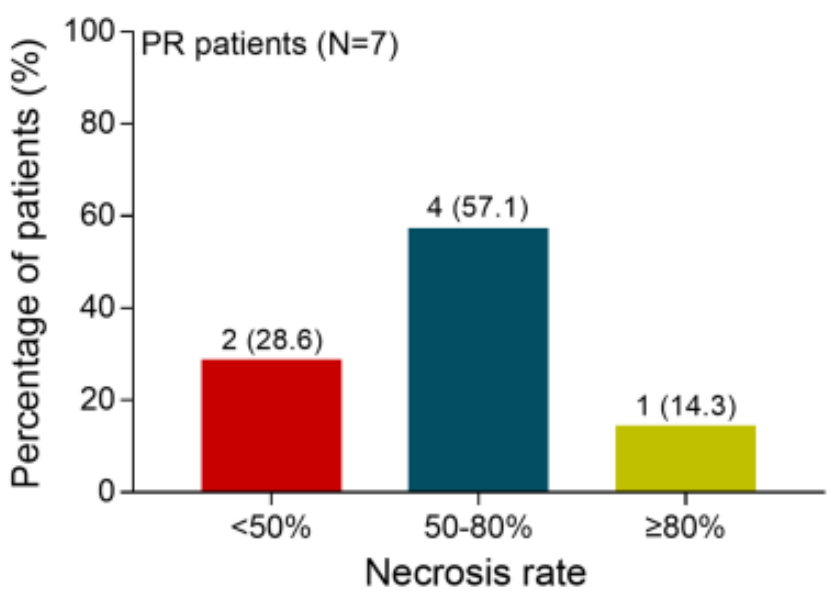

$\mathrm{D}$

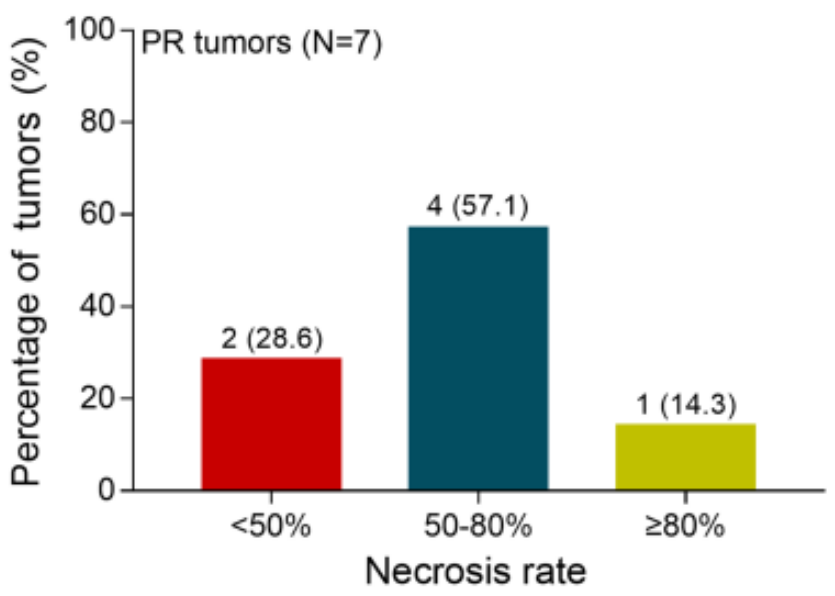

\section{Figure 1}

Treatment response after DEB-TACE treatment at one month A: The percentage of patients achieved clinical response in total patients; $\mathrm{B}$ : The percentage of patients achieved different necrosis rate in PR patients; C: The percentage of tumors achieved Clinical response in total tumors; D: The percentage of patients achieved different necrosis rate in PR tumors.

A

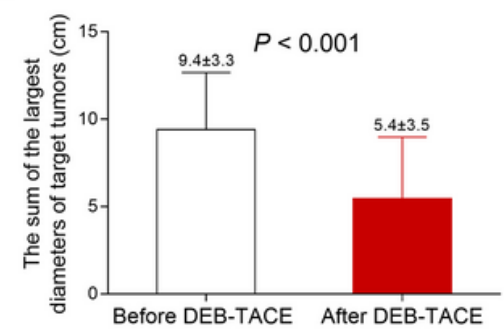

B

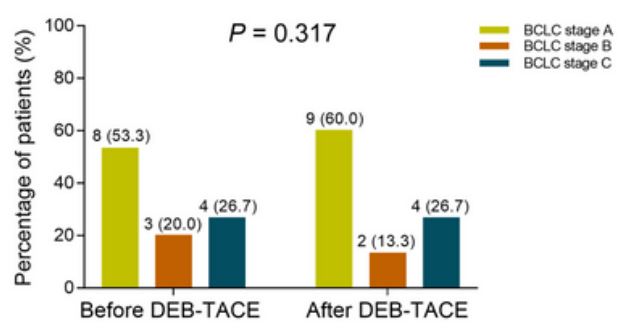

C

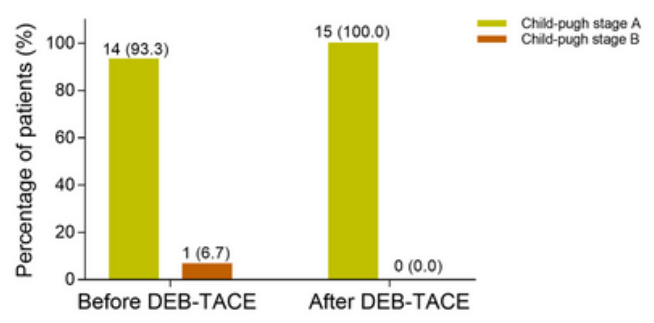

Figure 2

Tumor diameters, BCLC stage and Child-pugh stage before and after DEB-TACE A: Comparison of tumor diameters before and after DEB-TACE; B: Comparison of BCLC stage before and after DEB-TACE; C: 
Comparison of Child-pugh stage before and after DEB-TACE.

A

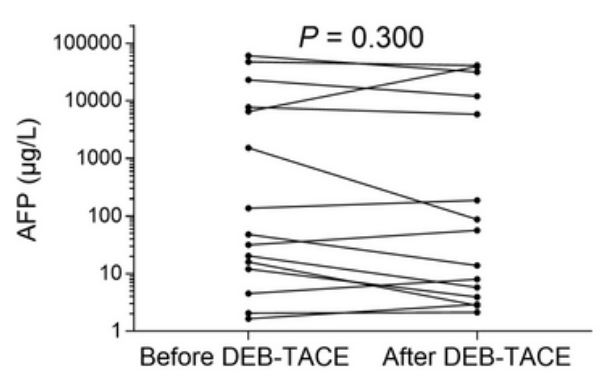

B

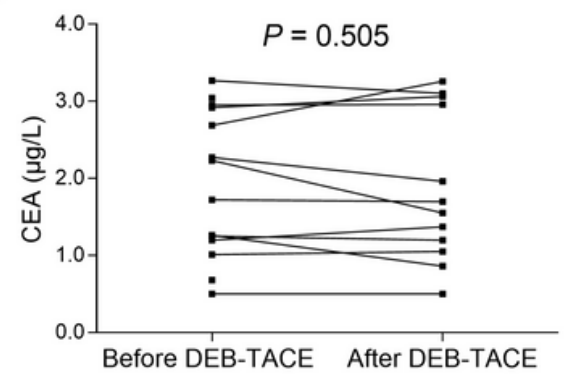

C

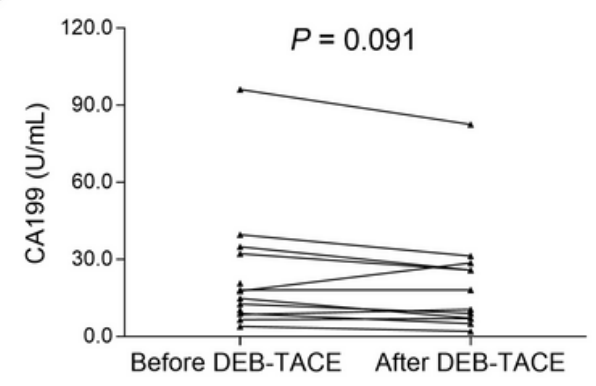

Figure 3

Tumor markers before and after DEB-TACE A: Comparison of serum AFP concentration before and after DEB-TACE; B: Comparison of serum CEA concentration before and after DEB-TACE; C: Comparison of serum CA191 concentration before and after DEB-TACE.

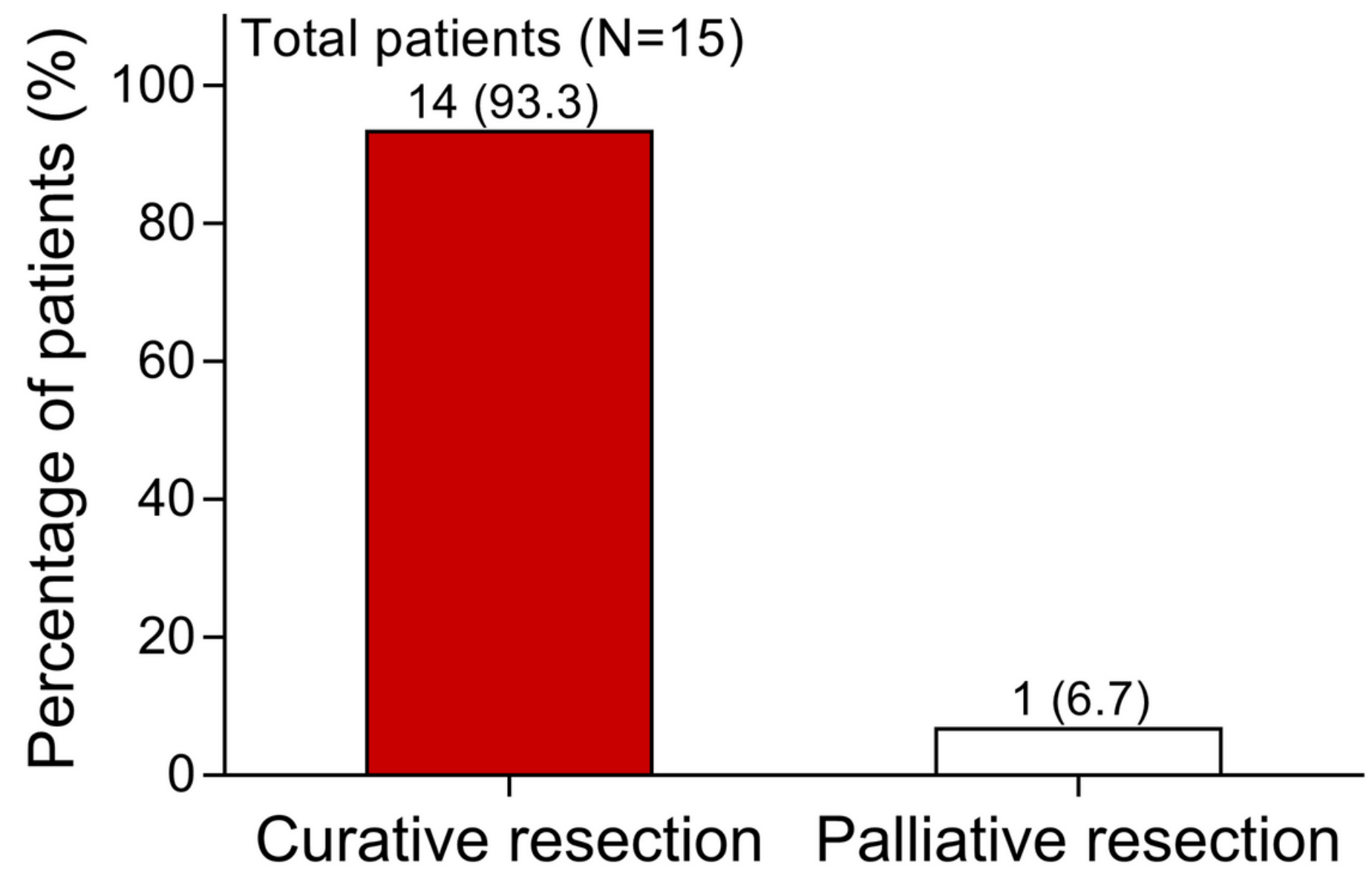

Figure 4

Operation types The percentage of patients achieved curative resection or palliative resection in total patients; 


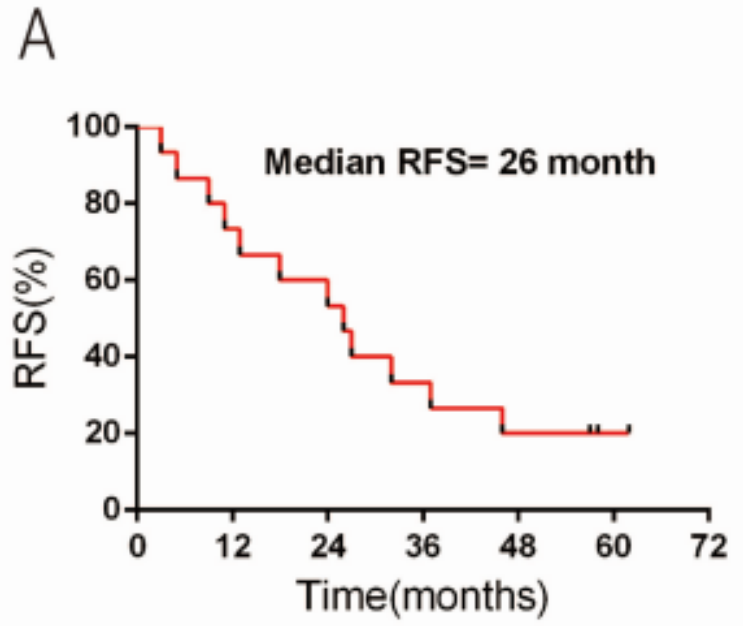

B

\section{Figure 5}

Survival A: The patients' RFS analyzed by Kaplan-Meier Survival curve; B: The patients' OS analyzed by Kaplan-Meier Survival curve.

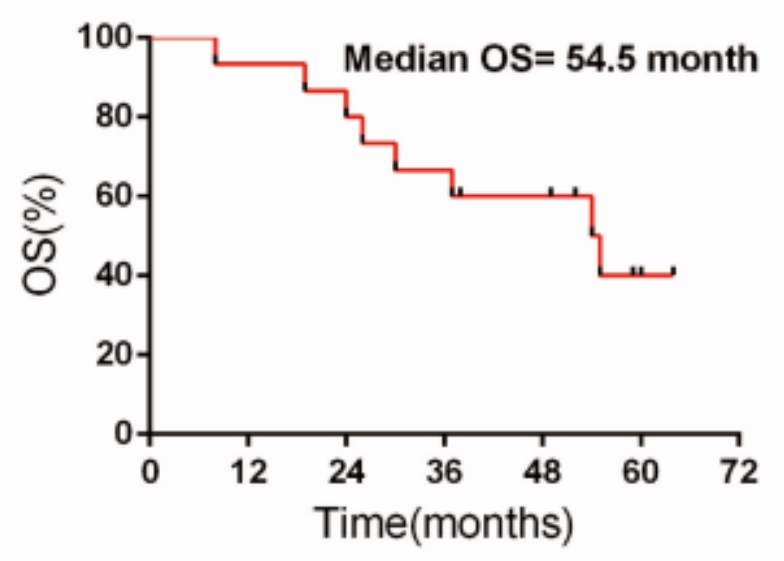

\section{Figure 6}

Typical Case report A: Representative images of tumor by abdominal computed tomography scan before DEB-TACE treatment; B: Representative images of DEB-TACE treatment; C: Representative images of Hepatic arteriography during DEB-TACE; D: Representative images of tumor Abdominal computed tomography scan after DEB-TACE treatment; E-F: Representative images of tumor during resection; G: Representative images of resected tumor; $\mathrm{H}-\mathrm{I}$ : Representative images of tumor pathological examination. 